\title{
Opportunistic Physical Design for Big Data Analytics
}

\author{
Jeff LeFevre $^{+\dagger}$ Jagan Sankaranarayanan* Hakan Hacıgümüş ${ }^{*}$ \\ Junichi Tatemura* Neoklis Polyzotis ${ }^{+}$Michael J. Carey ${ }^{\%}$ \\ ${ }^{*}$ NEC Labs America, Cupertino, CA ${ }^{+}$University of California, Santa Cruz ${ }^{\%}$ University of California, Irvine \\ \{jlefevre,alkis\}@cs.ucsc.edu, \{jagan,hakan,tatemura\}@nec-labs.com, mjcarey@ics.uci.edu
}

\begin{abstract}
Big data analytical systems, such as MapReduce, perform aggressive materialization of intermediate job results in order to support fault tolerance. When jobs correspond to exploratory queries submitted by data analysts, these materializations yield a large set of materialized views that we propose to treat as an opportunistic physical design. We present a semantic model for UDFs that enables effective reuse of views containing UDFs along with a rewrite algorithm that provably finds the minimum-cost rewrite under certain assumptions. An experimental study on real-world datasets using our prototype based on Hive shows that our approach can result in dramatic performance improvements.
\end{abstract}

\section{INTRODUCTION}

Data analysts have the crucial task of analyzing the ever increasing volume of data that modern organizations collect in order to produce actionable insights. As expected, this type of analysis on big data is highly exploratory in nature and involves an iterative process: the data analyst starts with an initial query over the data, examines the results, then reformulates the query and may even bring in additional data sources, and so on [9]. Typically, these queries involve sophisticated, domain-specific operations that are linked to the type of data and the purpose of the analysis, e.g., performing sentiment analysis over tweets or computing network influence. Because a query is often revised multiple times in this scenario, there can be significant overlap between queries. There is an opportunity to speed up these explorations by reusing previous query results either from the same analyst or from different analysts performing a related task.

MapReduce (MR) has become a de-facto tool for this type of analysis. It offers scalability to large datasets, easy incorporation of new data sources, the ability to query right away without defining a schema up front, and extensibility through user-defined functions (UDFs). Analyst queries are often written in a declarative query language, e.g., HiveQL or PigLatin, which are automatically translated to a set of MR jobs. Each MR job involves the materialization of intermediate results (the output of mappers, the input of reducers and the output of reducers) for the purpose of failure recovery. A typical Hive or Pig query will spawn a multi-stage job that will involve several such ma-

\footnotetext{
${ }^{\dagger}$ Work done when the author was at NEC Labs America

Permission to make digital or hard copies of all or part of this work for personal or classroom use is granted without fee provided that copies are not made or distributed for profit or commercial advantage and that copies bear this notice and the full citation on the first page. Copyrights for components of this work owned by others than ACM must be honored. Abstracting with credit is permitted. To copy otherwise, or republish, to post on servers or to redistribute to lists, requires prior specific permission and/or a fee. Request permissions from permissions@acm.org.

SIGMOD'14, June 22-27, 2014, Snowbird, UT, USA

Copyright is held by the owner/author(s). Publication rights licensed to ACM.

ACM 978-1-4503-2376-5/14/06 ...\$15.00.

http://dx.doi.org/10.1145/2588555.2610512
}

terializations. We refer to these execution artifacts as opportunistic materialized views.

We propose to treat these views as an opportunistic physical design and to use them to rewrite queries. The opportunistic nature of our technique has several nice properties: the materialized views are generated as a by-product of query execution, i.e., without additional overhead; the set of views is naturally tailored to the current workload; and, given that large-scale analysis systems typically execute a large number of queries, it follows that there will be an equally large number of materialized views and hence a good chance of finding a good rewrite for a new query. Our results indicate the savings in query execution time can be dramatic: a rewrite can reduce execution time by up to an order of magnitude.

Rewriting a query using views in the context of MR involves a unique combination of technical challenges that distinguish it from the traditional problem of query rewriting. First, the queries and views almost certainly contain UDFs, thus query rewriting requires some semantic understanding of UDFs. These MR UDFs for big data analysis are composed of arbitrary user-code and may involve a sequence of MR jobs. Second, any query rewriting algorithm that can utilize UDFs now has to contend with a potentially large number of operators since any UDF can be included in the rewriting process. Third, there can be a large search space of views to consider for rewriting due to the large number of materialized views in the opportunistic physical design, since they are almost free to retain (storage permitting).

Recent methods to reuse MR computations such as ReStore [6] and MRShare [21] lack any semantic understanding of execution artifacts and can only reuse/share cached results when execution plans are syntactically identical. We strongly believe that any truly effective solution will have to a incorporate a deeper semantic understanding of cached results and "look into" the UDFs as well.

Contributions. In this paper we present a novel query-rewrite algorithm that targets the scenario of opportunistic materialized views in an MR system with queries that contain UDFs. We propose a UDF model that has a limited semantic understanding of UDFs, yet enables effective reuse of previous results. Our rewrite algorithm employs techniques inspired by spatial databases (specifically, nearest-neighbor searches in metric spaces [12]) in order to provide a cost-based incremental enumeration of the huge space of candidate rewrites, generating the optimal rewrite in an efficient manner. Specifically, our contributions can be summarized as follows:

- A gray-box UDF model that is simple but expressive enough to capture a large class of MR UDFs that includes many common analysis tasks. The UDF model further provides a quick way to compute a lower-bound on the cost of a potential rewrite given just the query and view definitions. We provide the model and the types of UDFs it admits in Sections 3-4.

- A rewriting algorithm that uses the lower-bound to (a) gradually explode the space of rewrites as needed, and (b) only attempts a rewrite for those views with good potential to produce a low-cost 


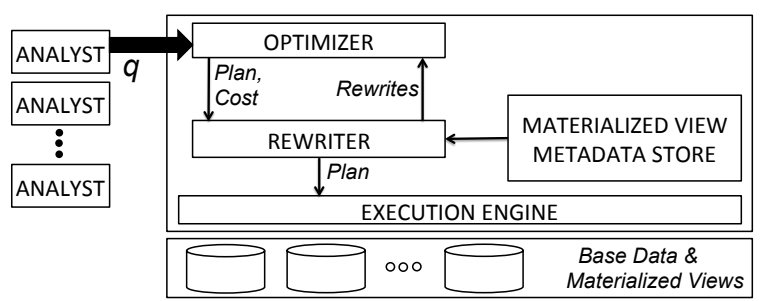

Figure 1: System diagram showing control flows.

rewrite. We show that the algorithm produces the optimal rewrite as well as finds this rewrite in a work-efficient manner, under certain assumptions. We describe this further in Sections 6-7.

- An experimental evaluation showing that our methods provide execution time improvements of up to an order of magnitude using real-world data and realistic complex queries containing UDFs. The execution time savings of our method are due to moving much less data and avoiding the high expense of re-reading data from raw logs when possible. We describe this further in Section 8.

\section{PRELIMINARIES}

Here we present the architecture of our system. We first briefly describe its components and how they interact with one another. We then provide the notations and problem definition.

\subsection{System Architecture}

Figure 1 provides a high level overview of our system and its components. Our system is built on top of Hive, and queries are written in HiveQL. Queries are posed directly over log data stored in HDFS. In Hive, MapReduce UDFs are given by the user as a series of Map or Reduce jobs containing arbitrary user code expressed in a supported language such as Java, Perl, Python, etc. To reduce execution cost, our system automatically rewrites queries based on the existing views. A query execution plan in Hive consists of a series of MR jobs, and each MR job materializes its output to HDFS. As Hive lacks a mature query optimizer and cannot cost UDFs, we implemented an optimizer based on the cost model from [21] and extended it to cost UDFs, as described later in Section 4.2.

During query execution, all by-products of query processing (i.e., the intermediate materializations) are retained as opportunistic materialized views. These views are stored in the system (space permitting) as the opportunistic physical design.

The materialized view metadata store contains information about the materialized views currently in the system such as the view definitions and standard data statistics used in query optimization. For each view stored, we collect statistics by running a lightweight Map job that samples the view's data. This constitutes a small overhead, but as we show experimentally in Section 8, this time is a small fraction of query execution time.

The rewriter, presented in Section 6, uses the materialize view metadata store to rewrite queries based on the existing views. To facilitate this, our optimizer generates plans with two types of annotations on each plan node: (1) the logical expression of its computation (Section 3.2) and (2) the estimated execution cost (Section 4.2).

The rewriter uses the logical expression in the annotation when searching for rewrites for each node in the plan. The expression consists of relational operators or UDFs. For each rewrite found during the search, the rewriter utilizes the optimizer to obtain an estimated cost for the rewritten plan.

\subsection{Notations}

$W$ denotes a plan generated by the query optimizer, which is represented as a DAG containing $n$ nodes, ordered topologically. Each node represents an MR job. We denote the $i^{t h}$ node of $W$ as $\operatorname{NODE}_{i}$, $i \in[1, n]$. The plan has a single sink that computes the result of the query; under the topological order assumption the sink is $\mathrm{NODE}_{n} . W_{i}$ is a sub-graph of $W$ containing $\operatorname{NODE}_{i}$ and all of its ancestor nodes. We refer to $W_{i}$ as one of the rewritable targets of plan $W$. As is standard in Hive, the output of each job is materialized to disk. Hence, a property of $W_{i}$ is that it represents a materialization point in $W$. In this way, materializations are free except for statistics collection. An outgoing edge from $\mathrm{NODE}_{k}$ to $\mathrm{NODE}_{i}$ represents data flow from $k$ to $i$. $V$ is the set of all opportunistic materialized views in the system.

We use COST $\left(\operatorname{NODE}_{i}\right)$ to denote the cost of executing the MR job at $\operatorname{NODE}_{i}$, as estimated by the query optimizer. Similarly, $\operatorname{CosT}\left(W_{i}\right)$ denotes the estimated cost of running the sub-plan rooted at $W_{i}$, which is computed as $\operatorname{COST}\left(W_{i}\right)=\sum_{\forall \operatorname{NODE}_{k} \in W_{i}} \operatorname{CosT}\left(\operatorname{NODE}_{k}\right)$.

We use $r_{i}$ to denote an equivalent rewrite of target $W_{i}$ iff $r_{i}$ uses only views in $V$ as input and produces an identical output to $W_{i}$, for the same database instance $D$. A rewrite $r^{*}$ represents the minimum cost rewrite of $W$ (i.e., target $W_{n}$ ).

\subsection{Problem Definition}

Given these basic definitions, we introduce the problem we solve in this paper.

Problem Statement. Given a plan $W$ for an input query $q$, and a set of materialized views $V$, find the minimum cost rewrite $r^{*}$ of $W$.

Our rewrite algorithm considers views in $V$ during the search for $r^{*}$. Since some views may contain UDFs, for the rewriter to utilize those views during its search, some understanding of UDFs is required. Next we will describe our UDF model and then present our rewrite algorithm that solves this problem.

\section{UDF MODEL}

Since big data queries frequently include UDFs, in order to reuse previous computation in our system effectively we desire a way to model MR UDFs semantically. If the system has no semantic understanding of the UDFs, then the opportunities for reuse will be limited - essentially the system will only be able to exploit cached results when one query applies the exact same UDF to the exact same input as a previous query. However, to the extent that we are able to "look into" the UDFs and understand their semantics, there will be more possibilities for reusing previous results. In this section we propose a UDF model that allows a deeper semantic understanding of MR UDFs. Our model is general enough to capture a large class of UDFs that includes classifiers, NLP operations (e.g., taggers, sentiment), text processors, social network (e.g., network influence, centrality) and spatial (e.g., nearest restaurant) operators. As an example, we performed an empirical analysis of two real-world UDF libraries, Piggybank [22] and DataFu [5]. Our model captures about $90 \%$ of the UDFs examined: 16 out of 16 Piggybank UDFs, and 30 out of 35 DataFu UDFs as detailed in [17]. Of course, we do not require the developer to restrict herself to this model; rather, to the extent a query uses UDFs that follow this model, the opportunities for reuse will be increased.

\subsection{Modeling a UDF}

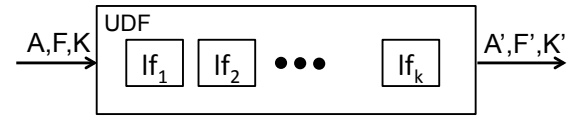

Figure 2: A UDF composed of local functions $\left(\boldsymbol{l} \boldsymbol{f}_{1}, \boldsymbol{l} \boldsymbol{f}_{2}, \cdots, \boldsymbol{l} \boldsymbol{f}_{k}\right)$, showing the end-to-end transformation of input to output.

We propose a model for UDFs that allows the system to capture a UDF as a composition of local functions as shown in Figure 2, where each local function represents a map or reduce task. The nature of the MR framework is that map-reduce functions are stateless and only operate on subsets of the input, i.e., a single tuple or a single group of tuples. Hence, we refer to these map-reduce functions as local func- 
tions. A local function can only perform a combination of the following three types of operations performed by map and reduce tasks.

1. Discard or add attributes, where an added attribute and its values may be determined by arbitrary user code

2. Discard tuples by applying filters, where the filter predicates may be performed by arbitrary user code

3. Perform grouping of tuples on a common key, where the grouping operation may be performed by arbitrary user code

The end-to-end transformation of a UDF is obtained by composing the operations performed by each local function $l f$ in the UDF. Our model captures the fine-grain dependencies between the input and output tuples in the following way.

The UDF input is modeled as $(A, F, K)$ where $A$ is the set of attributes, $F$ is set of filters previously applied to the input, and $K$ is the current grouping of the input, which captures the keys of the data. The output is modeled as $\left(A^{\prime}, F^{\prime}, K^{\prime}\right)$ with the same semantics. Our model describes a UDF as the transformation from $(A, F, K)$ to $\left(A^{\prime}, F^{\prime}, K^{\prime}\right)$ as performed by a composition of local functions using operation types (1) (2) (3) above. Figure 2 shows how to semantically model a UDF that takes any arbitrary input represented as $A, F, K$ and applies local functions to produce an output that is represented as $A^{\prime}, F^{\prime}, K^{\prime}$. Additionally, for any new attribute produced by a UDF (in the output schema $A^{\prime}$ ), its dependencies on the input (in terms of $A, F, K)$ are recorded as a signature along with the unique UDFname. Note that since the model only captures end-to-end transformations, for a UDF containing multiple internal jobs (e.g., the local functions in Figure 2), the system only retains the final output but not the intermediate results of local functions.

The model also captures UDFs that take multiple inputs, which is similar to the single input case shown in Figure 2. For example, a UDF that combines 2 inputs on a common key (similar to an equijoin) can be described in the following way. The inputs $\left\{A_{1}, F_{1}, K_{1}\right\}$ and $\left\{A_{2}, F_{2}, K_{2}\right\}$ produce an output $\left\{A_{J}, F_{J}, K_{J}\right\}$ such that: $A_{J}$ is the union of $A_{1}$ and $A_{2}, F_{J}$ is the conjunction of the filters $F_{1}, F_{2}$ and the join condition, and $K_{J}$ is $K_{1}$ union $K_{2}$ intersected with the join attributes. Note that the model only concerns itself with the end-to-end transformation of the inputs to the outputs, the actual implementation of an operator is not captured by the model.

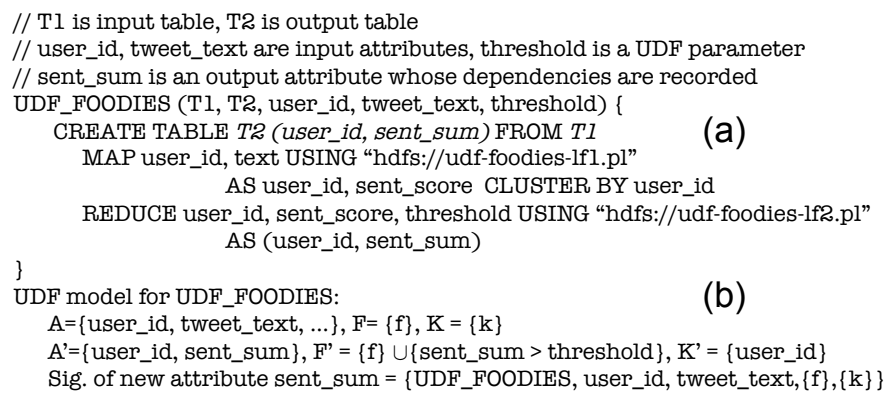

Figure 3: UDF_FOODIES a) implementation composed of two local functions, b) UDF model showing the end-to-end transformation of input to output.

As an example, consider UDF_FOODIES that applies a food sentiment classifier on tweets to identify users that tweet positively about food. An abbreviated HiveQL definition of the UDF is given in Figure 3(a) that invokes the following two local functions $\mathrm{lf}_{1}$ and $\mathrm{lf}_{2}$ written in a high-level language (Perl in this example). lf $_{1}$ : For each (user_id, tweet_text), apply the food sentiment classifier function that computes a sentiment value for each tweet about food. $\mathrm{lf}_{2}$ : For each user_id, compute the sum of the sentiment values to produce sent_sum, then filter out users with a total score greater than a threshold. Although the filter in this example is a simple com-
// Extract tweet_id, user_id and tweet_text from twitter log
CREATE TABLE $T 1$
SELECT tweet_id, user_id, tweet_text
FROM large_twitter_log;

// Create table T2 containing sent_sum for each user_id

UDF_FOODIES(T1, T2, user_id, tweet_text, 0.5)

// Join T1 and T2 to produce Result

CREATE TABLE Result

SELECT T2.user_id, Foo.count, T2.sent_sum FROM T2, (SELECT user id, COUNT( ${ }^{*}$ ) AS count FROM T1 GROUP BY user_id) AS Foo

WHERE Foo.user_id = T2.user_id AND Foo.count > 100;

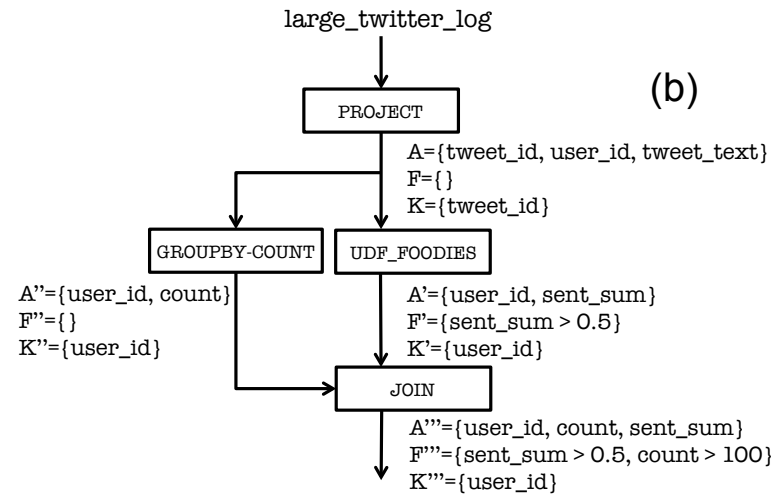

Figure 4: (a) Example query to obtain prolific foodies, and (b) corresponding annotated query plan.

parison operator, as noted above in (2) the filter expression may be a function containing arbitrary user code (i.e., a UDF) and may even contain a nesting of UDFs.

The two local functions correspond to arbitrary user code that perform complex text processing tasks such as parsing, word-stemming, entity tagging, and word sentiment scoring. Yet, the UDF model succinctly captures the end-to-end transformation of this complex UDF as shown in Figure 3(b). In the figure, the end-to-end transformation of UDF_FOODIES is captured by recording the changes made to the input $A, F$ and $K$ by the UDF functions that produces $A^{\prime}, F^{\prime}$ and $K^{\prime}$ using a simple notation. Furthermore, for the new attribute sent_sum in $A^{\prime}$, its dependencies on the subset of the inputs are recorded. We provide a more concrete example of the application of the UDF model in a HIVEQL query in Section 3.2. In this way, the model encodes arbitrary user-code representing a sequence of MR jobs, by only capturing its end-to-end transformations.

Our approach represents a gray-box model for UDFs, giving the system a limited view of the UDF's functionality yet allowing the system to understand the UDF's transformations in a useful way. In contrast, a white-box approach requires a complete understanding of how the transformations are performed, imposing significant overhead on the system. While with a black-box model, there is very little overhead but no semantic understanding of the transformations, limiting the opportunity to reuse any previous results.

\subsection{Applying the UDF Model and Annotations}

Having presented our model for UDFs, we now show how to use it to annotate a query plan that contains both UDFs and relational operators. In Figure 4(a), we show a query that uses Twitter data to identify prolific users who talk positively about food (i.e., "foodies"). The query is expressed in a simplified representation of HiveQL and applies UDF_FOODIES from Figure 3(a) that computes a food sentiment score (sent_sum) per user based on each user's tweets.

The HiveQL query is converted to an annotated plan as shown in Figure 4(b) by utilizing the UDF model of UDF_EOODIES as given in Figure 3(b). In addition to modeling UDFs, the three operations types (denoted as 1, 2, 3 above) can also be used to characterize standard relational operators such as select (2), project (1), join (2,3), group- 
by (3), and aggregation $(3,1)$. Joins in MR can be performed as a grouping of multiple relations on a common key (e.g., co-group in Pig) and applying a filter. Similarly, aggregations are a re-keying of the input (reflected in $K^{\prime}$ ) producing a new output attribute (reflected in $A^{\prime}$ ). These $A, F, K$ annotations can be applied to both UDFs and relational operations, enabling the system to automatically annotate every edge in the query plan.

Figure 4(b) shows the input to the UDF is modeled as $\langle A=\{$ user_id, tweet_id, tweet_text $\}, F=\emptyset, K=$ tweet_id $\rangle$. The output is $\left\langle A^{\prime}=\{\right.$ user_id, sent_sum $\}, F^{\prime}=$ sent_sum $>0.5, K^{\prime}=$ user_id $\rangle$. UDF_FOODIES produces the new attribute sent_sum whose dependencies are recorded (i.e., signature) as: $\langle A=\{$ user_id, tweet_text $\}, F=\emptyset, K=$ tweet_id, udf_name=UDF_FOODIES $\rangle$. Lastly, as shown in Figure 4(b), the output of the $\operatorname{UDF}\left(A^{\prime}, F^{\prime}, K^{\prime}\right)$ forms one input to the subsequent join operator, which in turn transforms its inputs to the final result.

This example shows how a query containing a UDF with arbitrary user code can be semantically modeled. The $A, F, K$ properties are straightforward and can be provided as annotations by the UDF creator with minimal overhead, or alternatively they may be automatically deduced using a static code analysis method such as [13], which is an emerging area of research. In this paper, we rely on the UDF creator to provide annotations, which is a one-time effort. From our experience evaluating the two UDF libraries [5,22] it took less than 10 minutes per UDF to examine the code and determine the annotations.

As noted earlier, our model is expressive enough to capture a large class of common UDFs. Two classes of UDFs not captured by our model, as noted in [17], are: (a) non-deterministic UDFs such as those that rely on runtime properties (e.g., current time, random, and stateful UDFs) and (b) UDFs where the output schema itself is dependent upon the input data values (e.g., pivot UDFs, contextual UDFs).

\section{USING THE UDF MODEL TO PERFORM REWRITES}

Our goal is to leverage previously computed results when answering a new query. The UDF model aids us in achieving this goal in three ways, as described in the following three sections. First, it provides a way to check for equivalence between a query and a view. Second, it aids in the costing of UDFs. Third, it provides a lower-bound on the cost of a potential rewrite.

\subsection{Equivalence Testing}

The system searches for rewrites using existing views and can test for semantic equivalence in terms of our model. We consider a query and a view to be equivalent if they have identical $A, F$ and $K$ properties. If a query and a view are not equivalent, our system considers applying transformations (sometimes referred to as compensations) to make the existing view equivalent to the query.

Here we develop the mechanics to test if a query $q$ (i.e., a target in the annotated plan) can be rewritten using an existing view $v$. Query $q$ can be rewritten using view $v$ if $v$ contains $q$. The containment problem is know to be computationally hard [2] even for the class of conjunctive queries, hence we make a first guess that only serves as a quick conservative approximation of containment. This conservative guess allows us to focus computational efforts toward checking containment on the most promising previous results and avoid wasting computational effort on less promising ones.

We provide a function $\operatorname{GuEss} \operatorname{Complete}(q, v)$ that performs this heuristic check. GuessComplete $(q, v)$ takes an optimistic approach, representing a guess that $v$ can produce a complete rewrite of $q$. This guess requires the following necessary conditions as described in [10] (SPJ) and [7] (SPJGA) that a view must satisfy to participate in a complete rewrite of $q$. (i) $v$ contains all attributes required by $q$; or contains all necessary attributes to produce those attributes in $q$ that are not in $v$

(ii) $v$ contains weaker selection predicates than $q$

(iii) $v$ is less aggregated than $q$

The function $\operatorname{GuessComplete}(q, v)$ performs these checks and returns true if $v$ satisfies the properties i-iii with respect to $q$. Note these conditions under-specify the requirements for determining that a valid rewrite exists, as they are necessary but not sufficient conditions. Thus the guess may result in a false positive, but will never result in a false negative. The purpose of $\operatorname{GuessComplete}(q, v)$ is to provide a quick way to distinguish between views that can possibly produce a rewrite from views that cannot. As rewriting is an expensive process, this helps to avoid examining views that cannot produce valid rewrites.

\subsection{Costing a UDF}

Given that our goal is to find a low cost rewrite for queries containing UDFs, we require a method of costing an MR UDF. We define the cost of a UDF as the sum of the cost of its local functions. Estimating the cost of a local function that performs any of the three operation types is complicated by two factors:

(a) Each operation type is performed by arbitrary user code, and thus can have varying complexity. For instance, although an NLP sentence tagger and a simple word-counter function perform the same operation type (discard or add attributes), they can have significantly different computational costs.

(b) There could be multiple operation types performed in the same local function, making it unrealistic to develop a cost model for every possible local function.

Due to these factors, we desire a conservative way to estimate the cost of a local function of varying complexity that may apply a sequence of operation types without knowing specifically how these operations interact with each other inside the local function.

Developing an accurate cost model is a general problem for any database system. In our framework, the importance of the cost model is only in guiding the exploration of the space of rewrites. For this reason, we appeal to an existing cost model from the literature [21], but slightly modify it to be able to cost UDFs. To this end, we extend the "data only" cost model in [21] in a limited way so that we are able to produce cost estimates for UDFs. Although this results in a rough cost estimate, experimentally we show that our cost model is effective in producing low cost rewrites (Section 8). The cost model we develop here is simple but works well in practice; however, an improved cost model may be plugged-in as it becomes available.

Recall that UDFs are composed of local functions, where each local function must be performed by a map task or a reduce task. The cost model in [21] accounts for the "data" costs (read/write/shuffle), and we augment it in a limited way to account for the "computational" cost of local functions. Since a UDF can encompass multiple jobs, we express the cost of each job as the sum of: the cost to read the data and apply a map task $\left(C_{m}\right)$, the cost of sorting and copying $\left(C_{s}\right)$, the cost to transfer data $\left(C_{t}\right)$, the cost to aggregate data and apply a reduce task $\left(C_{r}\right)$, and finally the cost to materialize the output $\left(C_{w}\right)$. Using this as a generic cost model, we first describe our approach toward solving (a) above by assuming that each local function only performs one instance of a single operation type. Then we describe our approach for (b), above.

For (a) we model the cost of the three operation types rather than each local function, which provides the baseline cost value for each operation type. Since there may be a high variation in the cost of a UDF's local functions, we apply a scalar multiplier to the baseline cost of $C_{m}, C_{r}$. To calibrate $C_{m}, C_{r}$ we take an empirical approach to estimate the scalar values. The first time the UDF is added to the 
system, we execute the UDF on a $1 \%$ uniform random sample of the input data to determine the scalar values. Due to data skew and onetime calibration, this may result in imprecise cost estimates. However, we do not preclude (a) recalibrating $C_{m}, C_{r}$ when the UDF is applied to new data, (b) a better sampling method if more is known about the data, and (c) periodically updating $C_{m}, C_{r}$ after executing the UDF on the full dataset.

For (b), since a local function performs an arbitrary sequence of operations of any type, it is difficult to estimate its cost. This would require knowing how the different operations actually interact with one another, which requires a white-box approach. For this reason we desire a conservative way to estimate the cost of a local function, which we do by appealing to the following property of any cost model performing a set $S$ of operations.

Definition 1. Non-subsumable cost property: Let $\operatorname{Cost}(S, D)$ be defined as the total cost of performing all operations in $S$ on a database instance $D$. The cost of performing $S$ on $D$ is at least as much as performing the cheapest operation in $S$ on $D$.

$$
\operatorname{CosT}(S, D) \geq \min (\operatorname{CosT}(x, D), \forall x \in S)
$$

The gray-box model of the UDFs only captures enough information about the local functions to provide a cost corresponding to the least expensive operation performed on the input. We cannot use the most expensive operation in $S$ (i.e., $\max (\operatorname{CosT}(x, D), \forall x \in S)$ ), since this requires $\operatorname{Cost}\left(S^{\prime}, D\right) \leq \operatorname{Cost}(S, D)$, where $S^{\prime} \subseteq S$. The "max" requirement is difficult to meet in practice, which we can show using a simple example. Suppose $S$ contains a filter with high selectivity, and a group-by with higher cost than the filter when considering these operations independently on database $D$. Let $S^{\prime}$ contain only groupby. Suppose that applying the filter before group-by results in few or no tuples streamed to group-by. Then applying group-by can have nearly zero cost and it is plausible that $\operatorname{Cost}\left(S^{\prime}, D\right)>\operatorname{Cost}(S, D)$.

The cost model utilizes the non-subsumable cost property in the following way. A local function that performs multiple operation types $t$ is given an initial cost corresponding to the generic cost of applying the cheapest operation type in $t$ on its input data. This initial value can then be scaled-up as described previously in our solution for (a).

\subsection{Lower-bound on Cost of a Potential Rewrite}

Now that we have a quick way to determine if a view $v$ can potentially produce a rewrite for query $q$, and a method for costing UDFs, we would like to compute a quick lower bound on the cost of any potential rewrite - without having to actually find a valid rewrite, which is computationally hard. To do this, we will utilize our UDF model and the non-subsumable cost property when computing the lower-bound. The ability to quickly compute a lower-bound is a key feature of our approach.

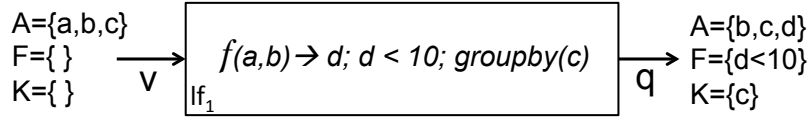

Figure 5: Synthesized UDF to perform the fix between a view $v$ and a query $q$.

Figure 5 provides an example showing a view $v$ and a query $q$ annotated as per the model, and a hypothetical local function $l f_{1}$, which we can use to compute a lower-bound as described next. View $v$ is given by attributes $\{a, b, c\}$ with no applied filters or grouping keys. Query $q$ is given by $\{b, c, d\}$, has a filter $d<10$, and has key $c$, where attribute $d$ is computed using $a$ and $b$. It is clear that $v$ is guessed to be complete with respect to $q$ because $v$ has all of the required attributes to produce those in $q$, and $v$ has weaker filters and grouping keys (i.e., is less aggregated) than $q$. Note that even though $v$ is guessed to be complete, grouping on $c$ may remove $a$ and $b$, which may render the creation of $d$ not possible; hence, it only a guess. However, since it does pass the
$\operatorname{GuessComplete}(q, v)$ test, we can then compute what we term as the fix for $v$ with respect to $q$. To determine the fix, we take the set difference between the attributes, filters, and keys $(A, F, K)$ of $q$ and $v$, which is straightforward and simple to compute. In Figure 5, the fix for $v$ with respect to $q$ is given by: a new attribute $d$; a filter $d<10$; and re-keying on $c$, as indicated in $l f_{1}$.

To produce a valid rewrite we need to find a sequence of local functions that "perform" the fix; these are the operations that when applied to $v$ will produce $q$. As this a known hard problem, we synthesize a hypothetical UDF comprised of a single local function that applies all operations in the fix (e.g., $l f_{1}$ in Figure 5). The cost of this synthesized UDF, which serves as an initial stand-in for a potential rewrite should one exist, is obtained using our UDF cost model. This cost corresponds to a lower-bound for any valid rewrite $r$. By the nonsubsumable cost property, the computational cost of this single local function is the cost of the cheapest operation in the fix. The benefit of the lower-bound is that it lets us cost views by their potential ability to produce a low-cost rewrite, without having to expend the computational effort to actually find one. Later we show how this allows us to consider views that are "more promising" to produce a low-cost rewrite before the "less promising" views are considered.

We define an optimistic cost function $\operatorname{OPTCOST}(q, v)$ that computes this lower-bound on any rewrite $r$ of query $q$ using view $v$ only if $\operatorname{GuessComplete}(q, v)$ is true. Otherwise $v$ is given OptCost of $\infty$, since in this case it cannot produce a complete rewrite, and hence the $\operatorname{Cost}$ is also $\infty$. The properties of $\operatorname{OptCost}(q, v)$ are that it is very quick to compute and

$$
\operatorname{OptCost}(q, v) \leq \operatorname{CosT}(r) .
$$

When searching for the optimal rewrite $r^{*}$ of $W$, we use OPTCOST to enumerate the space of the candidate views based on their cost potential, as we describe in the next section. This is inspired by nearest neighbor finding problems in metric spaces where computing distances between objects can be computationally expensive, thus preferring an alternate distance function (e.g., OPTCOST) that is easy to compute with the desirable property that it is always less than or equal to the actual distance.

\section{PROBLEM OVERVIEW FOR REWRIT- ING QUERIES CONTAINING UDFS}

Our UDF model enables reuse of views to improve query performance even when queries contain complex functions. However, reusing an existing view when rewriting a query with any arbitrary UDF requires the rewrite process to consider all UDFs in the system. The rewrite problem is known to be hard even when both the queries and the views are expressed in a language that only includes conjunctive queries $[10,19]$.

In our scenario, users are likely to include many UDFs in their queries. If the rewrite process were to consider every UDF as an operator in the rewrite language, searching for the optimal rewrite would quickly become impractical for any realistic workload and number of views. This is because the search space for finding a rewrite is exponential in both 1) the number of views in $V$ and 2) the number of operators (e.g., Relational and UDFs) considered by the rewrite process, which may include multiple applications of the same operator. For our rewrite algorithm, the worst case complexity is $O\left(n \cdot J^{|V|} \cdot k^{\left|L_{R}\right|}\right)$ where $n$ is the number of nodes in the plan $W, J$ is the maximum number of views that can participate in a rewrite, $|V|$ represents the number of views in the system, $k$ is maximum number of times that a particular operator can appear in a rewrite, and $\left|L_{R}\right|$ is the number of operators considered by the rewrite algorithm. For the experimental evaluation of our rewrite algorithm presented in Section 8, we set $J=4$ and $k=2$ for practical reasons. 
In our system, both the queries and the views can contain any arbitrary UDF, creating a potentially large number of UDFs in the system. Due to the complexity of the rewrite search process, in practice it is a good idea to limit the rewrite process to consider only a small subset of all UDFs in the system. For this reason, in our system the rewriter considers relational operators - select, project, join, group-by, aggregations (SPJGA), and a few of the most frequently used UDFs, which increases the possibility of reusing previous results. Selecting the right subset of UDFs to include in the rewrite process is an interesting open problem that must consider the tradeoff between the added expressiveness of the rewrite process versus the additional exponential cost incurred to search for rewrites.

A naive solution is to search for the optimal rewrite only for target $W_{n}$. However, (a) even if a rewrite is found for $W_{n}$, there may be a cheaper rewrite of $W$ using a rewrite found for a different target $W_{i}$, and (b) if one cannot find a rewrite for $W_{n}$, one may be able to find a rewrite at a different target $W_{i}$. The source of this problem is that $W_{n}$ may contain a UDF that is not included in the set of rewrite operators, and hence search process cannot be restricted only to $W_{n}$. For example, a rewrite for $W_{n}$ can be expressed by composing a rewrite $r_{i}$ for a target $W_{i}$ with the remaining nodes in $W$ indicated by $\operatorname{NODE}_{i+1} \cdots \operatorname{NODE}_{n}$. The composition of this rewrite could be cheaper than the rewrite found at $W_{n}$, thus the search process for the optimal rewrite must happen at all $n$ targets in $W$.

A better solution is to independently search for the best rewrite at each of the $n$ targets of $W$, and then use a dynamic programming solution to choose a subset among these to obtain the optimal rewrite $r^{*}$. One drawback of this approach is that there is no way of early terminating the search at a particular target since each search is independent. Hence, the search at one target does not inform the search at another. For instance, the algorithm may have searched for a long time at a target $W_{i}$ only to find an expensive rewrite, when it could have found a better (lower-cost) rewrite at an upstream target $W_{i-1}$ more quickly had it known to look there first.

The approach we take in this paper, called BFREWRITE, remedies these two shortcomings of the dynamic programming approach by (1) using the lower bound function OPTCOST introduced in Section 4.3 to guide the search process at each target, and (2) using results from the search process at one target to guide the search at the other targets. First, after finding a rewrite $r$ with cost $c$ at a target $W_{i}$, there is no need to continue searching for rewrites at $W_{i}$ if the OPTCOST of the the remaining unexplored space at $W_{i}$ is greater than $c$. Second, $r$ and $c$ can be used to prune the search space at other targets in $W$ by composing a rewrite of $W_{n}$ using $r$ and the remaining nodes (e.g., $\left.\operatorname{NODE}_{i+1} \cdots \operatorname{NODE}_{n}\right)$ in $W$.

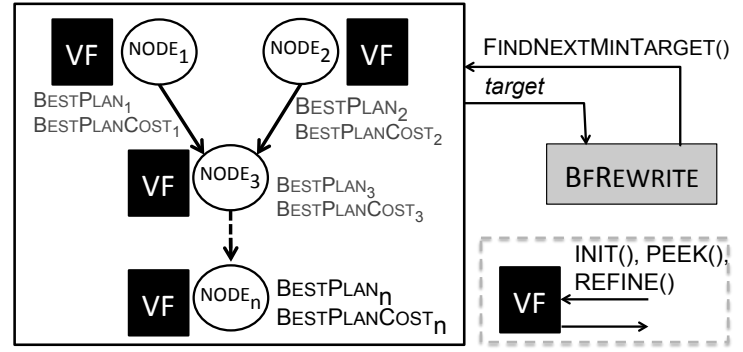

Figure 6: High level overview of the BFREWRITE algorithm.

Figure 6 provides a high-level overview of our BFREWRITE algorithm with plan $W$ represented as a DAG. Each node is associated with an instance of the VIEWFINDER module (VF), which is represented as a black box alongside described below. Additionally, each node stores its best rewrite found so far along with its cost. BFREWRITE interacts with this DAG using a function that identifies the next target to continue the rewrite search. On the right side of the figure, the interface to the black box VIEWFINDER is shown, which implements 3 simple primitives. Using this setup, the BFREWRITE algorithm performs a search for the globally optimal rewrite of W. There are 3 main components to the BFREWRITE algorithm.

1. VIEWFINDER at each target implements three operations - INIT sets up the initial search space of candidate views, ordering the available views by their OPTCOST; PEEK provides the OPTCOST of the next potential rewrite at the target; and REFINE which incrementally grows the space and attempts to find a rewrite of the target. This constitutes the local search at each target.

2. BFREWrite's FindNextMinTarget interface queries the DAG to identify the next target to explore. This constitutes the global search among the targets in $W$.

3. When a low-cost rewrite is found at a target, it is propagated to the remaining targets in $W$ by updating their best plan and its cost (BestPlan and BestPlanCost). This constitutes the update mechanism that coordinates the search of all targets in $W$.

These three components represent the global logic of BFREWRITE that explores the rewrite search space, at each step deciding the next target to explore. For each local search, the termination condition is that the remaining views to be examined (PEEK) have a lower bound cost that is greater than the best rewrite found so far. For the global search, the termination condition is that none of the targets has a potential of producing a lower cost rewrite of $W_{n}$ than the best one found so far. Note that due to the propagation, a node's best plan and cost do not necessarily correspond to the best rewrite found by the VIEWFINDER at that particular node, but could be a composition of rewrites found at other nodes.

In the next two sections, we provide the details of these components and the process outlined above. We first describe BFREWRITE in Section 6.1, which is the main driver of the rewrite search process, and is shown in Algorithm 1 and Algorithm 2. The mechanism to propagate the best rewrite is given in Algorithm 3. Then in Section 7 we describe the details of the VIEWFINDER component that is utilized as black box in the figure above.

\section{BEST-FIRST REWRITE}

The BFREWRITE algorithm produces a rewrite of $W$ that can be composed of rewrites found at multiple targets in $W$. The computed rewrite $r^{*}$ has provably the minimum cost among all possible rewrites in the same class. Moreover, the algorithm is work-efficient: even though $\operatorname{Cost}\left(r^{*}\right)$ is not known a-priori, it will never examine any candidate view with OPTCOST higher than the optimal cost $\operatorname{CosT}\left(r^{*}\right)$. To be work efficient, the algorithm must choose wisely the next candidate view to examine. As we will show below, the OptCost functionality plays an essential role in choosing the next target to refine. Intuitively, the algorithm explores only the part of the search space that is needed to provably find the optimal rewrite. We prove that BFREWRITE finds $r^{*}$ while being work-efficient in Section 6.2.

\subsection{The BFREWRITE Algorithm}

Algorithm 1 presents the main BFREWRITE function. In lines 26, BFREWRITE initializes a VIEWFINDER at each target $W_{i}$ and sets BESTPLAN $i$ and BESTPLANCOST $i$ to be the original plan and its cost. In lines 7-10, it repeats the following procedure: Invoke FindNextMinTarget (described in Algorithm 2) to choose the next best target to continue the search, which returns $\left(W_{i}, d\right)$, indicating that target $W_{i}$ can potentially produce a rewrite with a lower bound cost of $d$. Next, invoke REFINETARGET (described in Algorithm 2) which asks the VIEWFINDER to search for the next rewrite at target $W_{i}$. This continues until there is no target that can possibly im-

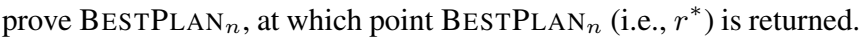

FINDNEXTMINTARGET in Algorithm 2 identifies the next best target $W_{i}$ to be refined in $W$, as well as the minimum cost (OPTCOST) 

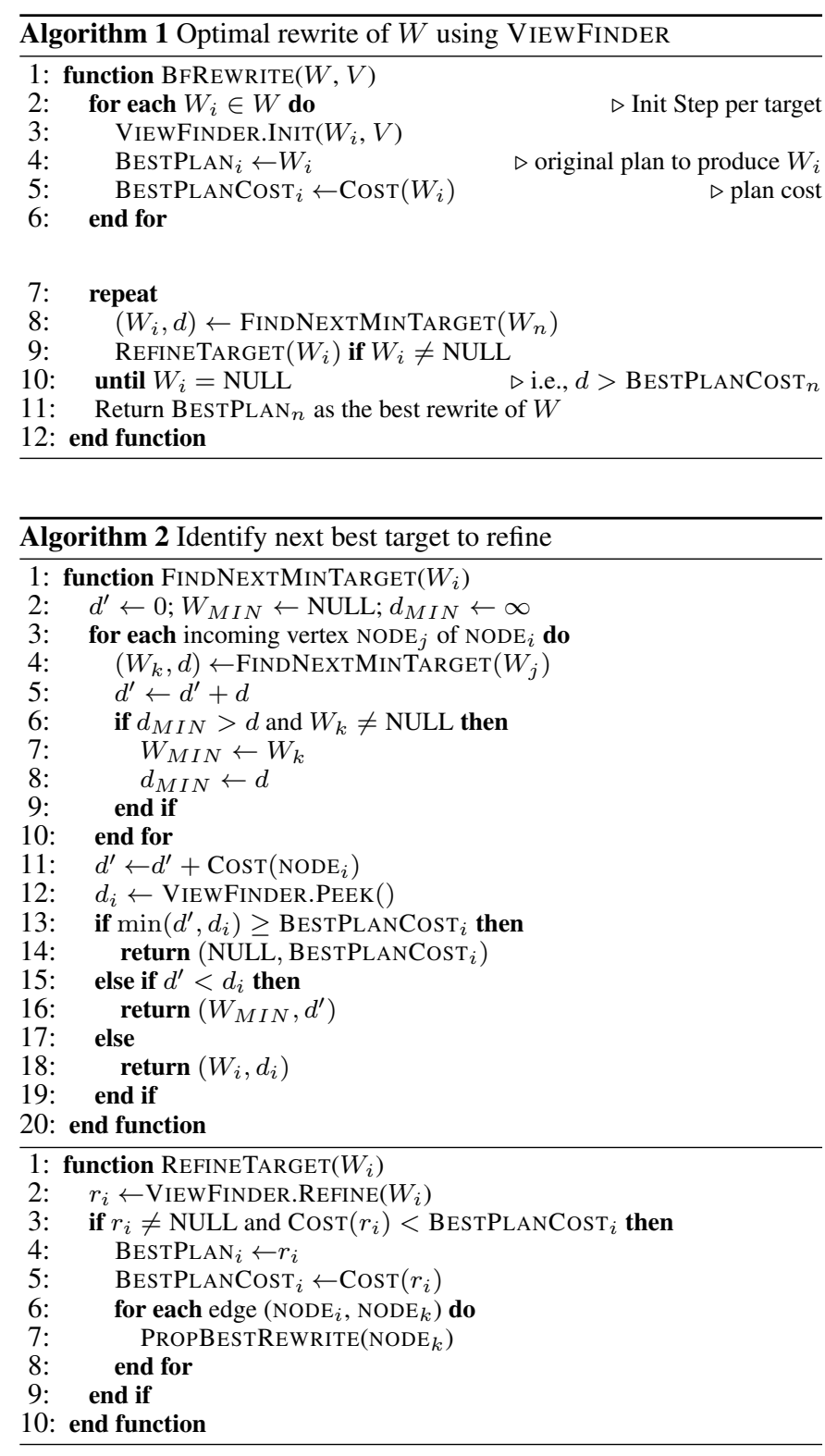

of a potential rewrite for $W_{i}$. There can be three outcomes of a search at a target $W_{i}$. Case 1: $W_{i}$ and all its ancestors cannot provide a better rewrite. Case 2: An ancestor target of $W_{i}$ can provide a better rewrite. Case 3: $W_{i}$ can provide a better rewrite. By recursively making the above determination at each target $W_{i}$ in $W$, the algorithm identifies the best target to refine next.

For a target $W_{i}$, the cost $d^{\prime}$ of the cheapest potential rewrite that can be produced by the ancestors of $\mathrm{NODE}_{i}$ is obtained by summing the VIEWFINDER.PEEK values at $\mathrm{NODE}_{i}$ 's ancestors nodes and the cost of $\operatorname{NODE}_{i}$ (lines 3-11). Note that we also record the target $W_{M I N}$ representing the ancestor target with the minimum OPTCOST candidate view (lines 6-9). Then $d_{i}$ is assigned to the next candidate view at $W_{i}$ using VIEWFINDER.PEEK (line 12).

Next the algorithm deals with the three cases outlined above. If both $d^{\prime}$ and $d_{i}$ are greater than or equal to BESTPLANCOST $i$ (case 1 ), there is no need to search any further at $W_{i}$ (line 13). If $d^{\prime}$ is less than $d_{i}$ (line 15), then $W_{M I N}$ is the next target to refine (case 2). Else (line 18), $W_{i}$ is the next target to refine (case 3 ).

Finally, REFINETARGET in Algorithm 2 describes the process of refining a target $W_{i}$. Refinement is a two-step process. In the first step it obtains a rewrite $r_{i}$ of $W_{i}$ from VIEWFINDER if one exists (line 2). The cost of the rewrite $r_{i}$ obtained by REFINETARGET is compared

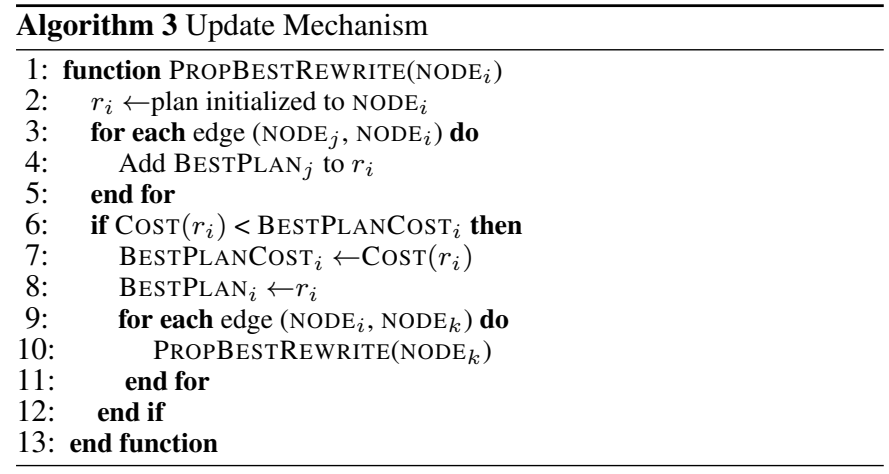

against the best rewrite found so far at $W_{i}$. If $r_{i}$ is found to be cheaper, the algorithm suitably updates BESTPLAN $i$ and BESTPLANCOST $i$ (lines 3-9). In the second step (line 7), the algorithm tries to compose a new rewrite of $W_{n}$ using $r_{i}$, through the recursive function given by PropBestRewrite in Algorithm 3. After this two-step refinement process, BESTPLAN ${ }_{n}$ contains the best rewrite of $W$ found so far.

PROPBESTREWRITE in Algorithm 3 describes the recursive update mechanism that pushes the new BESTPLAN $i$ downward along the outgoing nodes and towards $\operatorname{NODE}_{n}$. At each step it composes a rewrite $r_{i}$ using the immediate ancestor nodes of $\mathrm{NODE}_{i}$ (lines 2-5). It compares $r_{i}$ with BESTPLAN $i$ and updates BESTPLAN $i$ if $r_{i}$ is found to be cheaper (lines 6-12).

\subsection{Proof of Correctness and Work-Efficiency}

The following theorem provides the proof of correctness and the work-efficiency property of our BFREWRITE algorithm.

THEOREM 1. BFREWRITE finds the optimal rewrite $r^{*}$ of $W$ and is work-efficient.

PROOF. To ensure correctness, finding the optimal rewrite requires that the algorithm must not terminate before finding $r^{*}$. To ensure work-efficiency (defined earlier) requires that the algorithm should not examine any candidate views that cannot be possibly included in $r^{*}$.

A proof sketch by contradiction for a single target case (i.e., $n=1$ ) is as follows. Assume two cases: First, suppose that the algorithm found a candidate view $v$ resulting in a rewrite $r$, while the candidate view $v^{*}$, which produces the optimal rewrite $r^{*}$, is not considered before terminating even though $\operatorname{CosT}(r)>\operatorname{CosT}\left(r^{*}\right)$. Second, the algorithm examined a candidate view $v^{\prime}$ with $\operatorname{OpTCOST}\left(v^{\prime}\right)>$ $\operatorname{cost}\left(r^{*}\right)$. We can then show both these cases are not possible, proving that BFREWRITE finds $r^{*}$ in a work-efficient manner. The full proof for this single target case, which is then extended to the multi-target case, is provided in the extended version [17].

\section{VIEWFINDER}

The key feature of VIEWFINDER is its OPTCOST functionality that enables it to incrementally explore the the space of rewrites using the views in $V$. As noted earlier in Section 4.1, rewriting queries using views is known to be a hard problem. Traditionally, methods for rewriting queries using views for SPJG queries use a two stage approach $[1,10]$. The pruning stage determines which views are relevant to the query, and among the relevant views, those that contain all the required join predicates are termed as complete otherwise they are called partial solutions. This is typically followed by a merge stage that joins the partial solutions using all possible equijoin methods to form additional relevant views. The algorithm repeats until only those views that are useful for answering the query remain.

We take a similar approach in that we identify partial and complete solutions, then follow with a merge phase. The VIEWFInDER considers candidate views $C$ when searching for rewrite of a target. $C$ includes views in $V$ as well as views formed by "merging" views in 
$V$ using a MERGE function, which is an implementation of a standard view-merging procedure (e.g., $[1,10])$. Traditional approaches begin merging partial solutions to create complete solutions, until no partial solutions remain. This "explodes" the space of candidate views exponentially up-front. In contrast, our approach gradually explodes the space, resulting in far fewer candidates views from being considered.

Additionally, with no early termination condition, existing approaches would need to explore the space exhaustively at all targets. The VIEWFINDER incrementally grows and explores only as much of the space as needed, frequently stopping and resuming the search as requested by BFREWRITE.

\subsection{The VIEWFINDER Algorithm}

The VIEWFINDER is presented in Algorithm 4. An instance of VIEWFINDER instantiated at each target, which is stateful; enabling it to start, stop, and resume the incremental searches at each target. The VIEWFINDER maintains state using a priority queue (PQ) of candidate views, ordered by OPTCOST. VIEWFINDER implements the INIT, PEEK, and REFINE functions.

The INIT function instantiates a VIEWFINDER with a query $q$ representing a target $W_{i}$, and all views in $V$ are added to PQ, which orders them by increasing OPTCOST. The PEEK function returns the head item in PQ. The REFINE function is invoked when BFREWRITE asks the VIEWFINDER to examine the next candidate view.

REFINE pops the head item $v$ out of PQ and generates a set of new candidate views $M$ by merging $v$ with those views previously popped from PQ which were stored in Seen. Note that Seen only contains candidate views that have an OPTCOST less than or equal to that of $v$. Critically, this results in an "on-demand" incremental growth of the candidate space as required by BFREWRITE, rather than performing a pre-explosion of the entire search space. A property of the new candidate views in $M$, which is required for the correctness of the algorithm, is that they have an OPTCOST greater than $v$, hence none of these views could have been examined before $v$. This property is provided as a theorem in the extended version [17]. All newly created views in $M$ are inserted into PQ and $v$ is then added to Seen.

The REFINE function next attempts to find a rewrite using view $v$ by invoking REWRITEENUM, described next. Given the computational complexity of finding valid rewrites, VIEWFINDER limits the invocation of the REWRITEENUM algorithm using two strategies. First, the expensive REWRITEENUM operation is only applied to the view at the head of PQ when requested by BFREWRITE. Second, it avoids applying REWRITEENUM on every candidate view unless it passes the GUESSCOMPLETE test as described in Section 4.3.

\subsection{Rewrite Enumeration}

The REWRITEENUM function searches for a valid rewrite of query $q$ using view $v$ that has passed the GuessComplete test. Since GUESSCOMPLETE can result in false positives, there is no guarantee that $v$ will produce a valid rewrite for $q$. However, if a rewrite exists, REWRITEENUM returns the rewrite and its cost as computed by the CosT function.

In searching for a rewrite, recall from Section 5 that the rewrite process considers relational operators SPJGA and a subset of the UDFs in the system. These are the only rewrite operators considered by REWRITEENUM. The rewrite process searches for equivalent rewrites of $q$ by applying compensations [29] to $v$ and then testing for equivalence against $q$. In our implementation of REWRITEENUM this is done by generating all permutations of the rewrite operators and testing for equivalence, amounting to a brute force enumeration of all possible rewrites that can be produced with compensations. This makes the case for the system to keep the set of rewrite operators small since this search process is exponential in the size of this set. However, when the rewrite operators are restricted to a fixed known set, it may suffice to examine a polynomial number of rewrite attempts as in [8] for

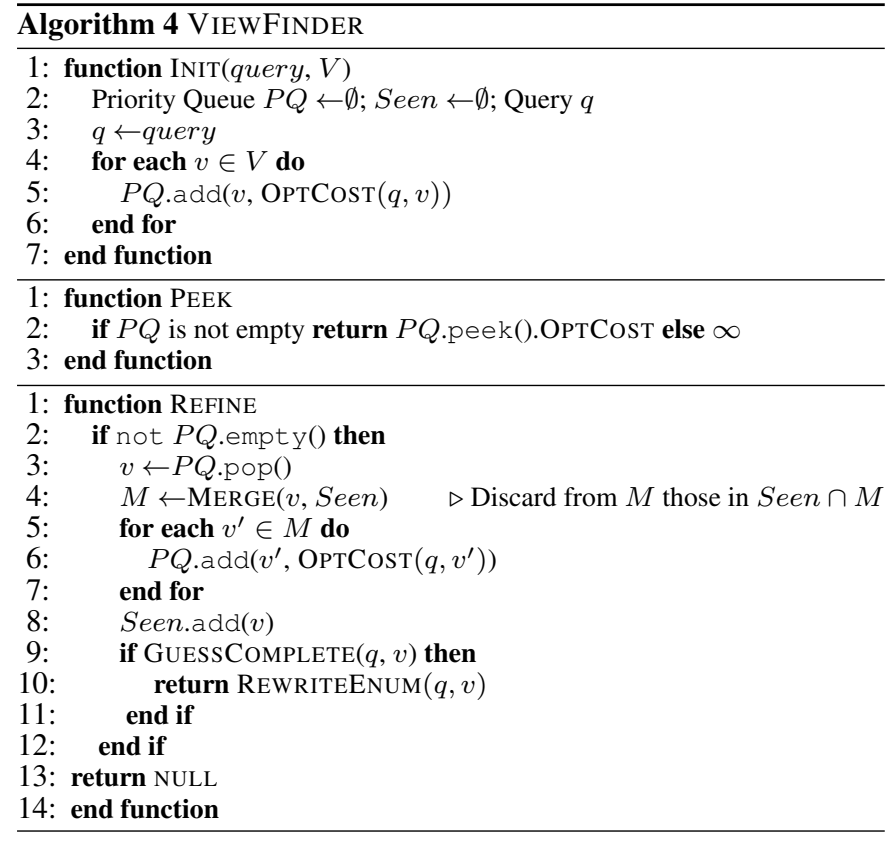

the specific case of simple aggregations involving group-bys. Such approaches are not applicable to our case as the system has the flexibility to add any UDF to the set of rewrite operators.

\section{EXPERIMENTAL EVALUATION}

In this section, we present an experimental study showing the effectiveness of BFREWRITE in finding low-cost rewrites of complex queries. First, we evaluate our methods in two scenarios. The query evolution scenario (Section 8.3.1) represents a user iteratively refining a query within a single session. This scenario evaluates the benefit that each new query version can receive from the opportunistic views created by previous versions of the query. The user evolution scenario (Section 8.3.2) represents a new user entering the system presenting a new query. This scenario evaluates the benefit a new query can receive from the opportunistic views previously created by queries of other "similar" users. Next, we evaluate the scalability (Section 8.3.3) of our rewrite algorithm in comparison to a competing approach. Lastly, we compare our method to cache-based methods (Section 8.3.4) that can only reuse previous results when they are identical.

\subsection{Query Workload}

We first provide some insights into the characteristics of exploratory processing on big data and then describe the workload from [16] that we adopted for use in this paper. Recent work [3,4,24] examines MR queries "in the wild" on production and research clusters that were utilized by data scientists or other advanced users for up to a year. Additional work $[11,14,27,28]$ provides further insights into big data analytical queries. A key finding of [4] is that there is a need for better benchmarks to capture the use cases for MR queries that perform interactive analysis on big data. Below we summarize the main findings from our literature review.

1. Users spend time revising and improving exploratory queries [14, 24], and thus queries near the end of an exploratory session tend to represent higher-quality and more complex versions of earlier queries [14].

2. Many studies note that complex analysis on big data frequently include UDFs [11,27,28].

3. Queries frequently incorporate multiple datasets [24] with a majority of queries (65\% in [24]) accessing three or more. 

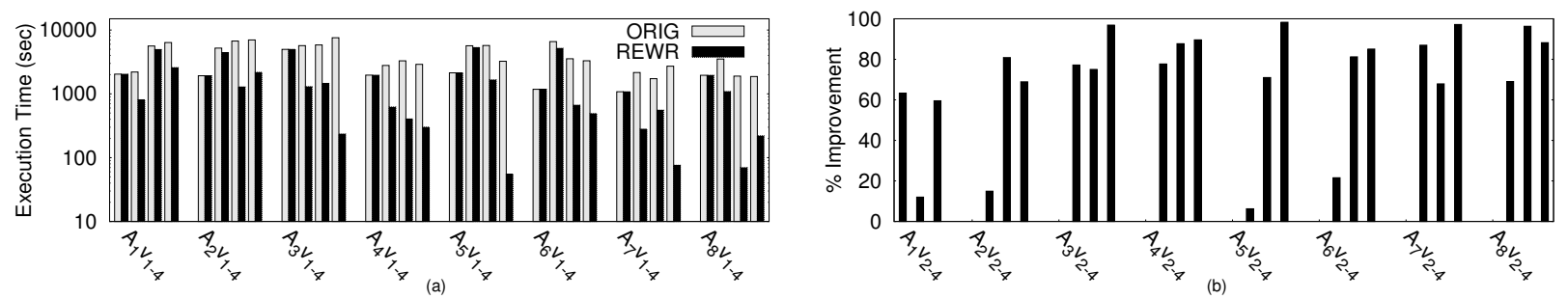

Figure 7: Query Evolution comparisons for (a) execution time (log-scale), and (b) execution time improvement.
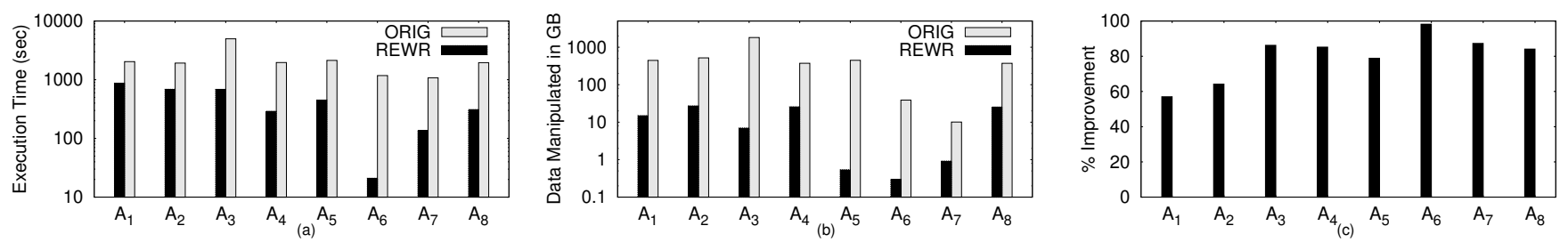

Figure 8: User Evolution comparisons for (a) execution time (log-scale), (b) data moved, and (c) execution time improvement.

Both [24] and [3] note that users frequently re-access their data and there can be significant benefits from caching. A majority of jobs involve data re-accesses with many occurring within 1 hour (50\% [3] and up to $90 \%$ [24]). These frequent re-access patterns make a strong case for a method such as BFREWRITE.

The experimental workload from [16] contains 32 queries on three datasets that simulate 8 analysts $A_{1}-A_{8}$ who write complex exploratory analytical queries for business marketing scenarios. Each query uses at least one of 10 unique UDFs. The workload uses three real-world datasets: A Twitter log (TWTR) of user tweets, a Foursquare $\log$ (4SQ) of user check-ins, and a Landmarks log (LAND) containing locations of interest. Many queries begin by accessing only one or two datasets, but subsequent revisions use all three datasets. Each of the 8 analysts poses 4 versions of a query, representing the initial query followed by three subsequent revisions made during data exploration and hypothesis testing. Hence, there is some overlap expected between subsequent versions of a query. The queries are longrunning with many operations, and executing the queries with Hive created 17 opportunistic materialized views per query on average.

Since each query in the workload has multiple versions, we use $A_{i} v_{j}$ to denote Analyst $i$ executing version $j$ of her query. Since there are 4 versions of each query, $A_{i} v_{j+1}$ represents a revision of $A_{i} v_{j}$. Below is a high-level description of query $A_{1} v_{1}$ and $A_{1} v_{2}$, taken from [16].

EXAMPLE 1. Analyst1 $\left(A_{1}\right)$ wants to identify a number of "wine lovers" to send them a coupon for a new wine being introduced in a local region.

Query $A_{1} v_{1}$ : (a) From TWTR, apply UDF-CLASSIFY-WINESCORE on each user's tweets and group-by user to produce a winesentiment-score for each user and then threshold on wine-sentimentscore. (b) From TWTR, compute all pairs $\left\langle u_{1}, u_{2}\right\rangle$ of users that communicate with each other, assigning each pair a friendship-strengthscore based on the number of times they communicate and then threshold on the friendship-strength-score. (c) From TWTR, apply UDAFCLASSIFY-AFFLUENT on users and their tweets. Join results from (a), (b), (c) on user_id.

Query $A_{1} v_{2}$ : Revise the previous version by reducing the winesentiment-score threshold, adding new data sources (4SQ and LAND) to find the check-in counts for users that check-in to places of type wine-bar, then threshold on count, joining this result with the users found in the previous version. Queries $A_{1} v_{3}$ and $A_{1} v_{4}$ are similarly revised by changing the threshold parameters and requiring that a user's friends also have a high check-in count to wine-bars.

The performance of any method that reuses results from previous queries will obviously depend on the degree of "similarity" between queries. However, choosing a meaningful metric to compute the similarity between queries in the workload from [16] was not clear. While methods such as [14] characterize query similarity in terms of query text (FROM clause, WHERE clause, etc.), we found this did not directly correspond with result reusability. We observed this effect in a microbenchmark we performed based on revising queries, and report those results in the extended version of the paper [17].

\subsection{Experimental Methodology}

Our experimental system consists of 20 machines running Hive version 0.7.1 and Hadoop version 0.20.2. Each node has the same hardware: 2 Xeon $2.4 \mathrm{GHz}$ CPUs ( 8 cores), $16 \mathrm{~GB}$ of RAM, and exclusive access to its own disk (2TB SATA 2012 model). We use HiveQL as the declarative query language, and Oozie as a job coordinator. The MR UDFs are implemented in Java, Perl, and Python and executed using the HiveCLI. UDFs implemented in our system include a log parser/extractor, text sentiment classifier, sentence tokenizer, lat/lon extractor, word count, restaurant menu similarity, and geographical tiling, among others. All UDFs are annotated using the model as per the example annotations given in Section 3.2. For each UDF in the workload we calibrate its cost model using the procedure described in Section 4.2. We provide an additional experiment in the extended version [17] to show that although we calibrate our cost model only the first time the UDF is added, it is able to discriminate between good plans and really bad plans for the purpose of query rewriting.

Our experiments use over 1TB of data that includes 800GB of TWTR tweets, 250GB of 4SQ check-ins, and 7GB of LAND containing 5 million landmarks. The identity of a user (user_id) is common across the TWTR and 4SQ logs, while the identity of a landmark (location_id) is common across 4SQ and LAND.

We report the following metrics for all experiments. Experiments on query execution time report both the original execution time of the query in Hive, labelled as ORIG, and the execution time of the rewritten query, labelled as REWR. The reported time for REWR includes the time to run the BFREWRITE algorithm, the time to execute the rewritten query, and any time spent on statistics collection. Experiments on the runtime of rewrite algorithms report the total time used by the algorithm to find a rewrite of the original query using the views in the system. For these experiments, BFR denotes our BFREWRITE rewriting algorithm, and DP represents a competing approach based on dynamic programming. DP does not use OPTCOST, and searches exhaustively for rewrites at every target. DP then rewrites a query by applying a dynamic programming solution to choose the best subset of rewrites found at each target. We note that both algorithms produce identical rewrites (i.e., $r^{*}$ ). The primary comparison metric for BFR and DP is algorithm runtime. In addition, we report results for two sec- 

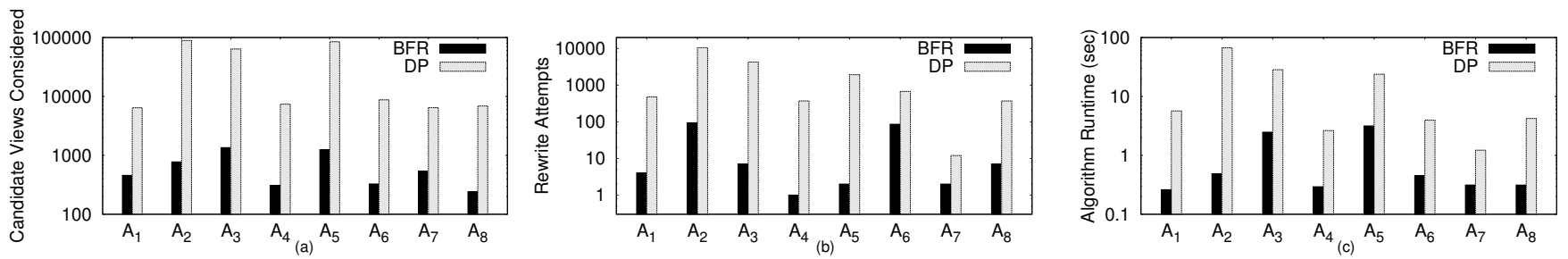

Figure 9: Algorithm comparisons for (a) candidate views considered, (b) rewrite attempts, and (c) Algorithm runtime (log-scale).

ondary metrics: the number of candidate views examined during the search for rewrites, and the number of valid rewrites attempted and produced during the search process. These correspond to the candidate space explored and rewrites attempted before identifying $r^{*}$.

\subsection{Experimental Results}

\subsubsection{Query Evolution}

In this experiment, for each analyst $A_{i}$, query $A_{i} v_{1}$ is executed followed by query $A_{i} v_{2}, A_{i} v_{3}$, and $A_{i} v_{4}$, applying BFREWRITE each time to rewrite the new query using the opportunistic views generated by the previous query versions. Before each Analyst $A_{i}$ begins, all views are dropped from the system. This experiment creates a scenario where an analyst may benefit by reusing results from previous versions of their own query. Figure 7(a) shows the execution time of the original query (ORIG) and the rewritten query (REWR), while Figure 7(b) reports the corresponding percent improvement in execution time of REWR over ORIG for each query ( $v_{1}$ is not shown since the percent improvement is always zero). Figure 7(b) shows that REWR provides an overall improvement of $10 \%$ to $90 \%$; with an average improvement of $61 \%$ and up to an order of magnitude. As a concrete data point, $A_{5} v_{4}$ requires 54 minutes to execute ORIG, but only 55 seconds to execute the rewritten query (REWR). REWR has much lower execution time because it is able to take advantage of the overlapping nature of the queries, e.g., version 2 has some overlap with version 1 . REWR is able to reuse previous results, providing significant savings in both query execution time and data movement (read/write/shuffle) costs.

\subsubsection{User Evolution}

In this experiment, each analyst (except one, a holdout analyst) executes the first version of their query. Then, we execute the first version of the holdout analyst's query (e.g., $A_{i} v_{1}$ ) after applying BFREWRITE to rewrite the holdout query using the opportunistic views generated by the previous queries. We then drop all views from the system and repeat using a different holdout analyst each time. This experiment creates a scenario where an analyst may benefit by reusing results from previous versions of other analysts' queries. Figure 8(a) shows the execution time for REWR and ORIG for each different holdout analyst along the $\mathrm{x}$-axis, while Figure $8(\mathrm{~b})$ shows the corresponding data manipulated (read/write/shuffle) in GB. These data statistics are automatically collected and reported by Hadoop and include the amount of data read from HDFS, moved across the network, and written to HDFS. These results demonstrate that the execution time of REWR is always lower than ORIG, and the data manipulated shows similar trends. The percentage improvement in execution time is given in Figure 8(c) which shows REWR results in an overall improvement of about $50 \%-90 \%$. Of course, these results are workload dependent but they show that even when several analysts query the same data sets while testing different hypothesis, our approach is able to find some overlap and hence benefit from previous results.

Table 1: Improvement in execution time of $A_{5} v_{3}$ as more analysts become present in the system.

\begin{tabular}{|l|l|l|l|l|l|l|l|}
\hline Analysts added & 1 & 2 & 3 & 4 & 5 & 6 & 7 \\
\hline Improvement & $0 \%$ & $73 \%$ & $73 \%$ & $75 \%$ & $89 \%$ & $89 \%$ & $89 \%$ \\
\hline
\end{tabular}

As an additional experiment for user evolution, we first execute a single analyst's query $\left(A_{5} v_{3}\right)$ with no opportunistic views in the system, to create a baseline execution time. Then we "add" another analyst by executing all four versions of that analyst's query, which creates new opportunistic views. Then we re-execute $A_{5} v_{3}$ and report the execution time improvement over the baseline, and repeat this process for the other remaining analysts. We chose $A_{5} v_{3}$ as it is a complex query that uses all three logs. Table 1 reports the execution time improvement after each analyst is added, showing that the improvement increases when more opportunistic views are present in the system.

\subsubsection{Algorithm Comparisons}

We first compare BFR to DP in terms of the number of candidate views considered, the number of times the algorithm attempts a rewrite, and the algorithm runtime in seconds. We use the user evolution scenario from the previous experiment, where there were approximately 100 views in the system when each holdout analyst's query was executed. Figure 9(a) shows that even though both algorithms find identical rewrites, BFR searches much less of the space than DP since it considers far fewer candidate views when searching for rewrites. Similarly, Figure 9(b) shows that BFR attempts far fewer rewrites compared to DP. This improvement can be attributed to GUESSCOMPLETE identifying the promising candidate views, and OPTCOST enabling BFR to incrementally explore the candidate views, thus applying REWRITEENUM far fewer times. Together, these contribute to BFR doing far less work than DP, which is reflected in the algorithm runtime shown in Figure 9(c). By growing the candidate views incrementally as needed (Figure 9(a)) and by controlling the number of times a rewrite is attempted (Figure 9(b)), BFREWRITE results in runtime significant savings since the both of these operations increase the search space exponentially.

We next test the scalability of BFR and DP by scaling up the number of views in the system from 1-1000 and report the algorithm runtime for both algorithms as they search for rewrites for one query $\left(A_{3} v_{1}\right)$. During the course of design and development of our system, we created and retained about 9,600 views; from these we discarded duplicate views as well as those views that are an exact match to the query (simply to prevent the algorithms from terminating trivially). In Figure 10, the x-axis reports the the number of views (views are randomly drawn from among these candidates), while the y-axis reports the algorithm run time (log-scale). DP becomes prohibitively expensive even when 250 views are present in the system, due to the exponential search space. BFR on the other hand scales much better than DP and has a runtime under 1000 seconds even when the system has 1000 views relevant to the given query. This is due to the ability of BFR to control the exponential space explosion and incrementally search the rewrite space in a principled manner.

While this runtime is not trivial, we note that these are complex queries involving UDFs that run for thousands of seconds. The amount of time spent to rewrite a query plus the execution time of the rewritten query is far less than the execution time of the original query. For instance, Figure 8(a) reports a query execution time of 451 seconds for $A_{5}$ optimized versus 2134 seconds for unoptimized. Even if the rewrite time for $A_{5}$ were 1000 seconds (it is actually 3.1 seconds here as seen in Figure 9(c)), the total execution time would still be $32 \%$ faster than the original query. 


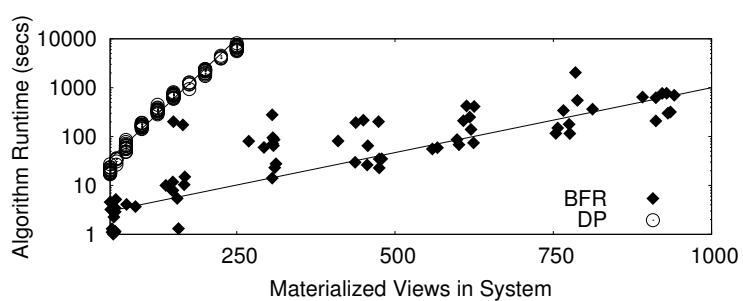

Figure 10: Runtime of BFR and DP for varying number of views.

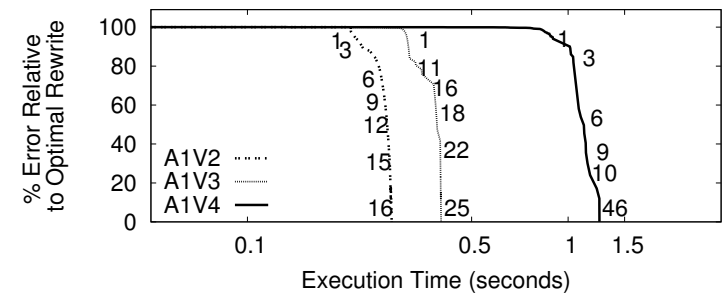

Figure 11: Execution time analysis of the quality of BFR's rewrite solutions found during its search for the optimal rewrite.

Finally, we show the effectiveness of OptCost in pruning the search space for BFR. Figure 11 shows the runtime behavior of BFREWRITE as it explores the space of rewrites in its search to identify the optimal rewrite. In this experiment, query $A_{1} v_{1}$ is first executed, producing a number of views. For each subsequent query $\left(A_{1} v_{2}, A_{1} v_{3}, A_{1} v_{4}\right)$, BFREWRITE searches for a rewrite given the views produced by the previous queries. The $\mathrm{x}$-axis reports the BFREWRITE's elapsed run time, and the y-axis reports the percent error relative to the optimal rewrite, in terms of cost. For each query the error begins at $100 \%$ (i.e., no rewrite has been found yet) and as BFREWRITE finds rewrites, the error is reduced until it reaches zero percent. At this point BFREWRITE has identified the optimal rewrite and can terminate the search (note that this is the same rewrite identified by the exhaustive DP algorithm). During the initial "flat" period for each query, BFREWRITE is growing the space of candidate views by examining views with the lowest OptCost. Since they failed to produce a rewrite, BFREWRITE begins merging them with views that have the next lowest OptCost. This phase represents BFREWRITE incrementally growing the space of candidate views, which is in contrast to an exhaustive approach (DP) that first grows the entire space of all possible candidate views before beginning to search for rewrites. The execution time for BFREWRITE increases slightly for the subsequent queries $A_{1} v_{3}$ and $A_{1} v_{4}$ since the execution of each subsequent query adds more views to the system.

In Figure 11, BFREWRITE finds the first and second valid rewrites for query $A_{1} v_{4}$ at about 0.9 seconds (indicated by the numbers 1 and 3 ) which reduces the error to $96 \%$ and $90 \%$ respectively. At shortly after 1 second, BFREWRITE finds valid rewrite number 46 which is the optimal rewrite and hence reduces the relative error to $0 \%$ and terminates. Two notable take-aways from Figure 11 are: (a) once BFREWRITE finds the first rewrite, it quickly converges to the optimal rewrite, and (b) when BFREWRITE finds the optimal rewrite and terminates for $A_{1} v_{4}$, it only had to find 46 rewrites before terminating, while the DP algorithm (not shown in figure) found 4656 rewrites. Similarly, BFREWRITE only had find 16 and 25 rewrites for $A_{1} v_{2}$ and $A_{1} v_{3}$ respectively, whereas DP found 66 and 323. This result illustrates a case when BFREWRITE can terminate early without examining all possible rewrites. These observations suggest that the OPTCOST is effective at pruning the search space for BFREWRITE.

\subsubsection{Comparison with Caching-based methods}

Next we provide a brief comparison of our approach with cachingbased methods (such as [6]) that perform only syntactic matching when reusing previous results. With this class of solutions, results can only be reused to answer a new query when their respective execution plans are identical, i.e., the new query's plan and the plan that produced the previous results must be syntactically identical. This means that if the respective plans differ in any way (e.g., different join orders or predicate push-downs), then reuse is not possible. For instance, with syntactic matching, a query that applies two filters in sequence $a, b$ will not match a view (i.e., a previous result) that has applied the same two filters in a different sequence $b, a$. In contrast, our BFR approach performs semantic matching and query rewriting. In this case, not only will BFR match $a, b$ with $b, a$, but it would also match the query to a view that only has filter $b$, by applying an additional filter $a$ during the rewrite process.

To represent the class of syntactic caching methods, we present a conservative variant of our approach that performs a rewrite only if a view and a query have identical $A, F, K$ properties as well as have identical plans. We term this variant BFR-SYNTACTIC. Figure 12 highlights the limitations of caching-based methods by repeating the query evolution experiment for Analyst $1\left(A_{1} v_{1}-A_{1} v_{4}\right)$. We first execute query $A_{1} v_{1}$ to produce opportunistic views, and then we apply both BFR and BFR-SYNTACTIC to queries $A_{1} v_{2}, A_{1} v_{3}$ and $A_{1} v_{4}$ and report the results in terms of query execution time improvement of the solutions produced by BFR and BFR-SYNTACTIC.

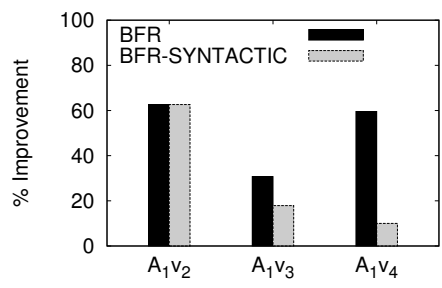

Figure 12 shows that both BFR and BFR-SYNTACTIC result in the same execution time improvement for $A_{1} v_{2}$. This is because both methods were able to reuse some of the (syntactically identical) views from the previous query. However, BFR-SYNTACTIC performs worse than BFR for query $A_{1} v_{3}$ and $A_{1} v_{4}$. This is because BFR-

Figure 12: Execution time improvement over ORIG.

SYNTACTIC was unable to find many views that were exact syntactic matches, whereas BFR was able to exploit additional views due to BFR's ability to reuse and re-purpose previous results through semantic query rewriting. Although this result is workload dependent, this example highlights the fact that while reusing identical results is clearly beneficial, our approach completely subsumes those that only reuse syntactically identical results: even when there are no identical views our method may still find a lowcost rewrite. To further illustrate this, we next perform an additional experiment after removing all identical views from the system before applying our BFREWRITE algorithm.

Table 2: Execution time improvement without identical views.

\begin{tabular}{|l|l|l|l|l|l|l|l|l|}
\hline & $A_{1}$ & $A_{2}$ & $A_{3}$ & $A_{4}$ & $A_{5}$ & $A_{6}$ & $A_{7}$ & $A_{8}$ \\
\hline BFR & $57 \%$ & $64 \%$ & $83 \%$ & $85 \%$ & $51 \%$ & $96 \%$ & $88 \%$ & $84 \%$ \\
\hline BFR-SYNTACTIC & $0 \%$ & $0 \%$ & $0 \%$ & $0 \%$ & $0 \%$ & $0 \%$ & $0 \%$ & $0 \%$ \\
\hline
\end{tabular}

Here we repeat the user evolution experiment after discarding from the system all views that are identical to a target in each of the holdout queries $\left(A_{1-8} v_{1}\right)$. Without these views, syntactic caching-based methods will not be able to find any rewrites, resulting in $0 \%$ improvement. Table 2 reports the percentage improvement for each analyst $A_{1}-A_{8}$ after discarding all identical views. This shows BFR continues to reduce query execution time dramatically even when there are no views in the system that are an exact match to the new queries. The performance improvements are comparable to the result in Figure 8(c) which represents the same experiment without discarding the identical views. Notably there is a drop for $A_{5}$ compared to the results reported in Figure 8(c) for $A_{5}$. This is because previously in Figure 8(c), $A_{5}$ had benefited from an identical view corresponding to a restaurant-similarity computation that it now has to recompute. The identical views discarded constituted only $7 \%$ of the view storage space in this experiment, indicating there are many other useful views. 
Given that analysts pose different but related queries, any method that relies solely on identical matching may have limited benefit.

\section{RELATED WORK}

Query Rewriting Using Views. There is a rich body of previous work on rewriting queries using views, but these only consider a restricted class of queries. Representative work includes the popular algorithm MiniCon [23], recent work [15] showing how rewriting can be scaled to a large number of views, and rewriting methods implemented in commercial databases [7,29]. However, in these works both the queries and views are restricted to the class of conjunctive queries (SPJ) or additionally include groupby and aggregation (SPJGA).

Our work differs in the following two ways: (a) We show how UDFs can be included in the rewrite process using our UDF model, which results in a unique variant of the rewrite problem when there is a divergence between the expressivity of the queries and that of the rewrite process; (b) Our rewrite search process is cost-basedOpTCOST enables the enumeration of candidate views based on their ability to produce a low-cost rewrite. In contrast, traditional approaches (e.g., $[7,23])$ typically determine containment first (i.e., if a view can answer a query) and then apply cost-based pruning in a heuristic way. This unique combination of features has not been addressed in the literature for the rewrite problem.

Online Physical Design Tuning. Methods such as [25] adapt the physical configuration to benefit a dynamically changing workload by actively creating or dropping indexes/views. Our work is opportunistic, and simply relies on the by-products of query execution that are almost free. However, view selection methods could be applicable during storage reclamation to retain only those views that provide maximum benefit.

Reusing Computations in MapReduce. Other methods for optimizing MapReduce jobs have been introduced such as those that support incremental computations [20], sharing computation or scans [21], and re-using previous results [6]. As shown in Section 8.3.4, our approach completely subsumes these methods.

Multi-query optimization (MQO). The goal of MQO [26] (and similar approaches [21]) is to maximize resource sharing, in particular common intermediate data, by producing a scheduling strategy for a set of in-flight queries. Our work produces a low-cost rewrite rather than a schedule for concurrent query plans.

\section{DISCUSSION AND CONCLUSION}

Big data analysis frequently includes exploratory queries that contain UDFs. Hence, to exploit previous results, a semantic understanding of UDF computation is required. In this work, we presented a gray-box UDF model that is simple but expressive enough to capture a large class of big data UDFs, enabling our system to effectively exploit prior computation. We also presented a rewrite algorithm that efficiently explores the large space of views.

Retaining opportunistic views within a limited storage space budget requires navigating the tradeoff between storage cost and query performance, which is equivalent to the view selection problem. During our experiments, accumulating all views for every query resulted in an additional storage space of only $2.0 \times$ the base data size $(\approx 2 \mathrm{~TB})$. The relatively small total size of all the views with respect to the log base data is due to several reasons. First, the logs are very wide, as they record a large number of attributes. However, a typical query only consumes a small fraction of these log attributes, which is consistent with observations in big data systems. Second, it is not uncommon for the log attributes to have missing values, since the data may be dirty or incomplete. For instance, in the Twitter log, a tweet may have missing location values, which a query may discard.

Developing a good view selection policy in this space is an interesting area of future work. One could consider access-based policies such as LRU and LFU, or cost-benefit based policies commonly used for physical design tuning. In the extended version [17] we show that the rewriter performs well even with a trivial storage reclamation policy, while in [18] we address a variant of this problem that considers several policies.

Acknowledgement: We thank Jeffrey Naughton for his insightful comments on the earlier versions of the paper. We also thank the anonymous SIGMOD reviewers for their useful feedback. J. LeFevre and N. Polyzotis were partially supported by NSF grant IIS-0447966 and a research grant from NEC labs.

\section{REFERENCES}

[1] S. Agrawal, S. Chaudhuri, and V. Narasayya. Automated selection of materialized views and indexes in SQL databases. In $V L D B, 2000$

[2] A. Chandra and P. M. Merlin. Optimal implementation of conjunctive queries in relational databases. In STOC, 1977.

[3] Y. Chen, S. Alspaugh, and R. Katz. Interactive analytical processing in big data systems: A cross-industry study of MapReduce workloads. $P V L D B, 5(12), 2012$.

[4] Y. Chen, A. Ganapathi, R. Griffith, and R. Katz. The case for evaluating MapReduce performance using workload suites. In MASCOTS, 2011.

[5] DataFu. http://data.linkedin.com/opensource/datafu.

[6] I. Elghandour and A. Aboulnaga. ReStore: Reusing results of MapReduce jobs. PVLDB, 5(6), 2012.

[7] J. Goldstein and P.-A. Larson. Optimizing queries using materialized views: A practical, scalable solution. In SIGMOD, 2001.

[8] S. Grumbach and L. Tininini. On the content of materialized aggregate views. In PODS, 2000

[9] H. Hacigümüs, J. Sankaranarayanan, J. Tatemura, J. LeFevre, and N. Polyzotis. Odyssey: A multi-store system for evolutionary analytics. $P V L D B, 6(11), 2013$.

[10] A. Halevy. Answering queries using views: A survey. VLDBJ, 10(4), 2001.

[11] J. M. Hellerstein, C. Ré, F. Schoppmann, D. Z. Wang, E. Fratkin, A. Gorajek, K. S. Ng, C. Welton, X. Feng, K. Li, et al. The MADlib analytics library: or MAD skills, the SQL. PVLDB, 5(12), 2012.

[12] G. Hjaltason and H. Samet. Index-driven similarity search in metric spaces. TODS, 28(4), 2003.

[13] F. Hueske, M. Peters, M. J. Sax, A. Rheinländer, R. Bergmann, A. Krettek, and K. Tzoumas. Opening the black boxes in data flow optimization. PVLDB, 5(11), 2012.

[14] N. Khoussainova, Y. Kwon, W.-T. Liao, M. Balazinska, W. Gatterbauer, and D. Suciu. Session-based browsing for more effective query reuse. In SSDBM, 2011.

[15] G. Konstantinidis and J. L. Ambite. Scalable query rewriting: a graph-based approach. In SIGMOD, 2011.

[16] J. LeFevre, J. Sankaranarayanan, H. Hacigümüs, J. Tatemura, and N. Polyzotis. Towards a workload for evolutionary analytics. In SIGMOD Workshop on Data Analytics in the Cloud (DanaC), 2013

[17] J. LeFevre, J. Sankaranarayanan, H. Hacıgümüş, J. Tatemura, N. Polyzotis, and M. J. Carey. Exploiting opportunistic physical design in large-scale data analytics. CoRR, abs/1303.6609, 2013.

[18] J. LeFevre, J. Sankaranarayanan, H. Hacıgümüş, J. Tatemura, N. Polyzotis, and M. J. Carey. MISO: Souping up big data query processing with a multistore system. In SIGMOD, 2014.

[19] A. Y. Levy, A. O. Mendelzon, and Y. Sagiv. Answering queries using views (extended abstract). In PODS, 1995.

[20] B. Li, E. Mazur, Y. Diao, A. McGregor, and P. Shenoy. A platform for scalable one-pass analytics using MapReduce. In SIGMOD, 2011.

[21] T. Nykiel, M. Potamias, C. Mishra, G. Kollios, and N. Koudas. MRShare: Sharing across multiple queries in MapReduce. $P V L D B$, 3(1-2), 2010

[22] PiggyBank. https://wiki.apache.org/pig/PiggyBank.

[23] R. Pottinger and A. Halevy. MiniCon: A scalable algorithm for answering queries using views. VLDBJ, 10(2), 2001

[24] K. Ren, Y. Kwon, M. Balazinska, and B. Howe. Hadoop's adolescence: An analysis of Hadoop usage in scientific workloads. PVLDB, 6(10), 2013.

[25] K. Schnaitter, S. Abiteboul, T. Milo, and N. Polyzotis. On-line index selection for shifting workloads. In ICDE, 2007

[26] T. Sellis. Multiple-query optimization. TODS, 13(1), 1988.

[27] A. Simitsis, K. Wilkinson, M. Castellanos, and U. Dayal. Optimizing analytic data flows for multiple execution engines. In SIGMOD, 2012

[28] R. S. Xin, J. Rosen, M. Zaharia, M. J. Franklin, S. Shenker, and I. Stoica. Shark: SQL and rich analytics at scale. In SIGMOD, 2013.

[29] M. Zaharioudakis, R. Cochrane, G. Lapis, H. Pirahesh, and M. Urata. Answering complex SQL queries using automatic summary tables. In SIGMOD, 2000 